\title{
EXACT SOLUTIONS OF TWO NONLINEAR EQUATIONS AND HYPERCIRCLE ESTIMATES
}

\author{
V. G. HART
}

(Received 25 October 1979)

\begin{abstract}
In a recent paper estimates of the solutions of two nonlinear differential equations were made by use of the hypercircle method. Here exact solutions are given which are compared with those estimates.
\end{abstract}

\section{Introduction}

In a recent paper [1] estimates for the solution of certain nonlinear differential equations were found by use of the hypercircle method. In this note it is pointed out that both examples considered in [1] admit exact solutions and comparison is made with the trial functions previously used. Of course, the general method described in [1] is still available for problems in which the exact solution is not known.

\section{Solution for example 1}

The first example concerned the nonlinear ordinary differential equation problem

$$
\left.\begin{array}{rl}
\frac{d^{2} \phi}{d x^{2}} & =\phi+\phi^{2} / 4, \quad-1<x<1, \\
\phi(-1) & =\phi(1)=1 .
\end{array}\right\}
$$

The solution of this problem is given in terms of Jacobian elliptic functions as follows. Multiplication of the equation in (1) by $d \phi / d x$ and integration leads to

$$
\frac{d \phi}{d x}=\left[\phi^{2}-\phi_{0}^{2}+(1 / 6)\left(\phi^{3}-\phi_{0}^{3}\right)\right],
$$


on using the evenness of the solution in $x$, and setting $\phi(0)=\phi_{0}$, as yet unknown. Previous results in [1] show that $\phi^{\prime}$ vanishes only at the origin in $[0,1]$, and that $\phi$ is monotonic increasing in this interval.

Separation of variables in (2) and integration leads to the solution

$$
\phi(x)=\phi_{0}-u_{1} s c^{2}\left(\lambda^{-1} 6^{-\frac{1}{2}} x \mid m\right)
$$

with the notation of [3], and where

$$
\left.\begin{array}{l}
u_{1,2}=-3\left(1+\phi_{0} / 2\right) \pm \frac{1}{2}\left[3\left(2-\phi_{0}\right)\left(6+\phi_{0}\right)\right]^{\frac{1}{2}} \\
\lambda^{-1}=\frac{1}{2}\left(-u_{2}\right)^{\frac{1}{2}} \text { and } m=\left(u_{1}-u_{2}\right) /\left(-u_{2}\right) .
\end{array}\right\}
$$

In order to satisfy the boundary conditions in (1), we require that $\phi_{0}$ satisfies

$$
\phi_{0}=1+u_{1} s c^{2}\left(\lambda^{-1} 6^{-\frac{1}{2}} \mid m\right) \equiv f\left(\phi_{0}\right)
$$

and $\phi_{0}$ must be found by numerical iteration. This can be a tedious process unless a good initial estimate of $\phi_{0}$ is available; happily this is provided by the previous estimate 0.6100 in [1]. Since $\left|f^{\prime}(0.6100)\right| \simeq 0.07$, the iteration $\phi_{r+1}=f\left(\phi_{r}\right)$ converges reasonably quickly to the value $\phi_{0}=0.60850$, and the comparison in Table 1 of the exact solution $\phi$ of (1) with our trial function $\phi_{1}$ of [1] can be made.

TABLE 1

Comparison of exact solution $\phi$ and trial function $\phi_{1}$ of example 1

\begin{tabular}{lcccccr}
\hline$x$ & 0.0 & \pm 0.2 & \pm 0.4 & \pm 0.6 & \pm 0.8 & \pm 1.0 \\
$\phi$ & 0.60850 & 0.62258 & 0.66557 & 0.73977 & 0.84918 & 1.0 \\
$\phi_{1}$ & 0.60100 & 0.61696 & 0.66484 & 0.74464 & 0.85636 & 1.0 \\
\hline
\end{tabular}

The mean-square error is found by numerical integration (Simpson's rule with $h=0.05$ ) :

$$
\left\langle\phi-\phi_{1}, \phi-\phi_{1}\right\rangle=\int_{-1}^{1}\left(\phi-\phi_{1}\right)^{2} d x=5.5 \times 10^{-5},
$$

so that the upper bound of $[1], E\left(\phi_{1}\right)=44 \times 10^{-5}$ is conservative, as could be expected with this simple function $\phi_{1}$.

Further, it can be remarked that the trial function $\phi_{1}$ of [1], found by minimizing the hypersphere radius,

$$
\phi_{1}=0.601+0.399 x^{2},
$$

is a close pointwise approximation in $[0,1]$ to the expansion of the exact solution (3) to the same order :

$$
\phi \simeq 0.6085+0.35053 x^{2}
$$




\section{Solution for example 2}

The second example in [1] was the nonlinear boundary-value problem in the plane

$$
\left.\begin{array}{c}
\nabla^{2} \phi=k e^{\phi} \quad \text { in } \quad V, \\
\phi=1 \text { on } B,
\end{array}\right\}
$$

where $V$ is the disc $r<1$, so that $B$ is given by $r=1$. Here $k$ is a positive real constant. There is a general solution available for the differential equation in (9) (with $k$ positive or negative) due to Liouville [2], and an alternative derivation of this appears in the appendix. It would clearly be of use if the domain $V$ were other than the unit disc, and a conformal map on the unit disc were employed.

However, since in (9) radial symmetry is assumed, there is merely an ordinary differential equation to be solved,

$$
\left(r \phi^{\prime}\right)^{\prime}=k r e^{\phi},
$$

where a dash denotes differentiation with respect to $r$. If we differentiate (10) again we get

$$
\left(r \phi^{\prime}\right)^{\prime \prime}=k e^{\phi}\left(1+r \phi^{\prime}\right)
$$

Setting

$$
v=1+r \phi^{\prime}
$$

and eliminating the exponential by means of (10), we find from (11) that

$$
r v^{\prime \prime}=v v^{\prime} \text {. }
$$

Next, using $v^{\prime \prime}=v^{\prime} d v^{\prime} / d v$, and noting that

$$
\frac{d}{d v}\left(r v^{\prime}\right)=1+r \frac{d v^{\prime}}{d v}
$$

we obtain from (13) that

$$
\frac{d}{d v}\left(r v^{\prime}\right)=v+1
$$

after division by $v^{\prime}$. Integration then gives

$$
r v^{\prime}=\frac{v^{2}}{2}+v+C
$$

and the condition $v=1$ at $r=0$ gives $C=-3 / 2$ since we assume $\phi^{\prime}$ finite in $[0,1]$.

Separation of variables and integration gives $v$ as a rational function of $r^{2}$; then, returning to $\phi^{\prime}$ by (12), another integration gives 


$$
\phi=\ln \left\{A\left(a^{2}-r^{2}\right)^{-2}\right\},
$$

where $A$ and $a$ are arbitrary constants.

Satisfaction of the differential equation and boundary condition in (9) requires that the constants satisfy the conditions

$$
A=8 a^{2} / k \text { and } e\left(1-a^{2}\right)^{2}=A .
$$

Solving the resulting quadratic equation for $a^{2}$ and selecting the root greater than unity, we then obtain the solution

$$
\phi(r)=\ln \left[\left(8 a^{2} / k\right)\left(a^{2}-r^{2}\right)^{-2}\right],
$$

with

$$
a^{2}=1+4 /(k e)+\left\{(1+4 /(k e))^{2}-1\right\}^{\frac{1}{2}} .
$$

Since $a^{2}$ is greater than unity the solution is continuous in $V$. We now compare it with the hypercircle estimate. Using the exact solution (16) we calculate the meansquare error $\left\langle\phi-\phi_{1}, \phi-\phi_{1}\right\rangle$ of the trial function

$$
\phi_{1}=1+\beta\left(r^{2}-1\right)
$$

used in [1]. A comparison with the upper bound $E\left(\phi_{1}\right)$ for this error is given in Table 2, and again $E\left(\phi_{1}\right)$ is conservative, but could of course be improved by using a more elaborate trial function.

TABLE 2

Mean-square errors and upper bounds, $E\left(\phi_{1}\right)$, example 2

\begin{tabular}{cccc}
\hline$k$ & $\beta$ & $10^{4}\left\langle\phi-\phi_{1}, \phi-\phi_{1}\right\rangle$ & $10^{4} E\left(\phi_{1}\right)$ \\
\hline 5 & 1.405 & 81.6 & 1641.5 \\
2 & 0.8065 & 9.69 & 166.6 \\
1 & 0.4926 & 1.49 & 22.17 \\
\hline
\end{tabular}

The pointwise approximation of $\phi_{1}$ to the power series approximation for

$$
\phi \simeq a+b r^{2}
$$

is not quite as good as in the case of example 1, but improves as $k$ decreases. For example, for $k=1$ we have

$$
\phi \simeq 0.5252+0.4227 r^{2} \text { and } \phi_{1}=0.5074+0.4926 r^{2} .
$$

\section{Acknowledgement}

The author expresses his thanks to Professor A. F. Pillow for suggesting the possibility of obtaining exact solutions to these problems and also for helpful discussions. 


\section{Appendix. Derivation of the solution to problem (9)}

Liouville's general solution [2] of the differential equation in (9) can be written

$$
\phi=\ln \left\{A F^{\prime}(z) \overline{F^{\prime}(z)} /[F(z) \overline{F(z)}+c]^{2}\right\},
$$

where $A$ and $c$ are arbitrary constants, $z=x+i y, F(z)$ is an arbitrary analytic function of $z$, and the bar denotes the complex conjugate. Both positive and negative values of the parameter $k$ in (9) are allowed.

The following derivation seems a little more direct than the original, and follows at the outset that of Weston [4]. The equation in (9) is

$$
\phi_{z \bar{z}}=(k / 4) e^{\phi},
$$

where subscripts denote partial derivatives. Differentiating this equation with respect to $z$ we find

$$
\phi_{z z \bar{z}}=\phi_{z \bar{z}} \phi_{z}
$$

having eliminated the exponential. Integration gives

$$
\phi_{z z}-\frac{1}{2} \phi_{z}^{2}=B_{1}(z)
$$

where $B_{1}$ is an analytic function of $z$. Next we differentiate (A2) with respect to $\bar{z}$ obtaining similarly

$$
\phi_{\bar{z} \bar{z}}-\frac{1}{2} \phi_{\bar{z}}^{2}=B_{2}(\bar{z})
$$

where $B_{2}$ is an antianalytic function of $\bar{z}$ (that is, $\partial B_{2} / \partial z=0$ ).

Now in (A4) set

$$
\phi_{z}=\frac{F^{\prime \prime}(z)}{F^{\prime}(z)}+G(z, \bar{z}),
$$

where $F$ is analytic, the dash denoting the derivative, and $F^{\prime}$ is supposed nonzero in $r<1$. We deduce that $G$ satisfies a Riccati equation

$$
G_{z}=\frac{F^{\prime \prime}}{F^{\prime}} G+\frac{1}{2} G^{2}
$$

Setting, as usual, $G=-2 h_{z} / h$, we solve (A7) for $G$ and find that

$$
\phi_{z}=F^{\prime \prime}(z) / F^{\prime}(z)-2 F^{\prime}(z) k(\bar{z}) /[F(z) k(\bar{z})+n(\bar{z})],
$$

with $k$ and $n$ arbitrary.

Similarly, from (A5) we may deduce that

$$
\phi_{\bar{z}}=\overline{F^{\prime \prime}(z)} / \overline{F^{\prime}(z)}-2 \overline{F^{\prime}(z)} s(z) /[\overline{F(z)} s(z)+p(z)],
$$


with $s$ and $p$ arbitrary. Finally, using $\phi_{z \bar{z}}=\phi_{z z}$, we find by comparison that $s(z)=F(z), k(\bar{z})=\overline{F(z)}$, and $n(\bar{z})=p(z)=c$, constant. The solution (A1) is thus established.

In the case of the problem in (9), we set $F(z)=z$ in (A1), and the constants $A$ and $c$ $\left(=-a^{2}\right)$ are determined as in Section 3.

\section{References}

[1]. A. M. Arthurs and V.G. Hart, "The method of the hypercircle for a class of nonlinear equations", $J$. Austral. Math. Soc. B 21 (1979), 75-83.

[2]. J. Liouville, "Sur l'équation aux différences partielles $\left[\partial^{2} /(\partial u \partial v)\right](\log \lambda) \pm \lambda / 2 a^{2}=0$ ", J. de Math. 18 (1853), 71-72.

[3]. L. M. Milne-Thomson, Jacobian elliptic function tables (Dover, 1950).

[4]. V. H. Weston, "On the asymptotic solution of a partial differential equation with an exponential nonlinearity", SIAM J. Math. Anal. 9 (1978), 1030-1053.

\section{Department of Mathematics}

University of Queensland

St. Lucia

Queensland 4067 